\title{
Egg and cholesterol intake and incident type 2 diabetes among French women
}

\author{
Martin Lajous ${ }^{1,2,3}$, Anne Bijon ${ }^{3,4}$, Guy Fagherazzi ${ }^{3,4}$, Beverley Balkau ${ }^{3,4}$, Marie-Christine Boutron-Ruault ${ }^{3,4}$ \\ and Françoise Clavel-Chapelon ${ }^{3,4 *}$ \\ ${ }^{1}$ Center for Research on Population Health, INSP (Instituto Nacional de Salud Pública), Cuernavaca, Mexico \\ ${ }^{2}$ Department of Epidemiology, Harvard School of Public Health, Boston, MA, USA \\ ${ }^{3}$ Center for Research in Epidemiology and Population Health (CESP), Institut National de la Santé et de la Recherche Médicale \\ (INSERM), Villejuif 94805, France \\ ${ }^{4}$ Paris-South University, Orsay 91400, France
}

(Submitted 22 September 2014 - Final revision received 10 June 2015 - Accepted 1 July 2015 - First published online 10 September 2015)

\section{Abstract}

Egg consumption is a major source of dietary cholesterol, a nutrient that may disrupt glucose metabolism. We prospectively evaluated the relation between egg consumption and cholesterol-intake and diabetes in 65364 French disease-free women who responded to a validated diet history questionnaire in 1993. Egg consumption included hardboiled eggs and eggs consumed in an omelette or a mixed dish, and dietary cholesterol was estimated using a French nutrient database. Over 14 years of follow-up, 1803 incident diabetes cases were identified through self-reports, supplementary questionnaires and drug reimbursement information. Multivariable Cox regression models were adjusted for age, education, menopause, menopausal hormone therapy, hypertension and hypercholesterolaemia, BMI, physical activity, smoking, alcohol, fruit, vegetables, processed red meat, coffee and sugar and artificially sweetened beverages. No association was observed between egg consumption and risk of type 2 diabetes. When comparing women who consumed at least five eggs per week with non-consumers, the multivariable hazard ratio (HR) was found to be 1.00 (95\% CI $0.78,1 \cdot 29$; across categories, $P_{\text {trend }}=0 \cdot 11$ ). Women in the highest quintile of dietary cholesterol had a $40 \%$ higher rate of diabetes compared with those in the lowest quintile (HR 1.40; 95\% CI 1.19, 1.63; across quintiles, $P_{\text {trend }}<0.0001$ ). A $100 \mathrm{mg}$ increase of dietary cholesterol per $4184 \mathrm{~kJ}$ (or $1000 \mathrm{kcal}$ ) was associated with a $14 \%$ increase in the risk of diabetes (HR 1.14; $95 \%$ CI 1.02, 1.26). In this large prospective cohort, we observed an association between dietary cholesterol and type 2 diabetes, but no association with egg consumption. In the absence of a clear underlying mechanism and potential residual confounding, these results should be interpreted with caution.

\section{Key words: Prospective studies: Diet: Eggs: Cholesterol: Type 2 diabetes}

The observation that high egg consumption is strongly associated with incident gestational diabetes suggests that egg consumption may disrupt glucose metabolism ${ }^{(1)}$. Eggs are a major source of cholesterol, and in animal models cholesterol intake disrupts glucose metabolism and increases circulating insulin $^{(2)}$. In humans, dietary cholesterol appears to affect type 2 diabetes risk ${ }^{(3)}$, and elevated cholesterol intake prior to pregnancy is associated with an increased risk for gestational diabetes $^{(4)}$. Few prospective studies have evaluated the relation between egg consumption and incidence of type 2 diabetes. Among middle-aged US adults, men and women who consumed at least one egg a day had a 58 and a $77 \%$ higher rate of diabetes compared with those who did not consume eggs ${ }^{(5)}$. This relation was not observed in a cohort of Adventists ${ }^{(6)}$, in older US adults ${ }^{(7)}$, or in two European ${ }^{(8,9)}$ and a Japanese ${ }^{(10)}$ population. Such inconsistency could be explained by differences in the distribution of egg consumption, in the variables used for adjustment for confounding, in the method of preparation or in the foods consumed in conjunction with eggs. Therefore, we investigated the relation between egg and cholesterol consumption and incident type 2 diabetes in a large prospective cohort of French women.

\section{Methods}

\section{Study population}

The E3N study (Etude Epidémiologique auprès des femmes de la Mutuelle Générale de l'Education Nationale) is a French prospective study of 98995 women born between 1925 and 1950 and affiliated to a health insurance plan that covers mostly teachers and their spouses that began in 1990. E3N is the French component of the European Prospective Investigation into Cancer and Nutrition. Cohort members have returned mailed questionnaires on reproductive and lifestyle information and on newly diagnosed diseases in 1992, 1993, 1994, 1997, 2000, 2002,

\footnotetext{
Abbreviation: HR, hazard ratio.
}

* Corresponding author: F. Clavel-Chapelon, email francoise.clavel@gustaveroussy.fr 
2005 and 2008. Loss to follow-up has been low (3\%), and average follow-up in each questionnaire cycle has been $83 \%$. All women signed an informed consent form in compliance with the French National Commission for Computerized Data and Individual Freedom. In 1993, 74154 women responded to a follow-up questionnaire and a validated self-administered diet history questionnaire $^{(11)}$. We excluded those with an unrealistic energy consumption ( $n$ 1366), no follow-up ( $n$ 720), prevalent diabetes ( $n$ 1576), diabetes with no date of diagnosis ( $n$ 81), prevalent cancer or CVD ( $n$ 4950) and women who reported following a diet for diabetes $(n$ 97). The final study population comprised 65364 women.

\section{Dietary and covariate assessment}

Between 1993 and 1995, dietary data were collected using a previously validated 208-item self-administered diet history questionnaire, with eleven categories of frequency: never or less than once a month; one, two or three times a month; and one to seven times a week. Total egg consumption was based on the number and frequency of consumption of hardboiled eggs and of eggs consumed in omelettes or mixed dishes. Nutrient intakes, including that of dietary cholesterol, were calculated using a food composition table derived from a French national database. The validity and reproducibility of the dietary questionnaire was previously assessed in a sample of 119 women who had completed two diet history questionnaires and twelve monthly 24-h dietary recalls over a 1-year period ${ }^{(11)}$. The correlation coefficient between the dietary questionnaire and the 24-h recalls was 0.49 for eggs and 0.40 for dietary cholesterol. Education was assessed in 1990 at cohort recruitment with the following categories: no studies, primary-school certificate, secondary-school certificate, high-school certificate (baccalaureate) to 2-year post-high-school education, 3 to 4 years of post-high-school education and 5 or more years of post-high-school education. We defined universityeducated individuals as those who responded to the last two categories. For other covariates, we used information from a questionnaire sent to participants along with the dietary assessment. Treated hypertension and hypercholesterolaemia, and smoking were based on self-reports. Menopausal status was determined using information on last menstruation, the presence of hot flushes, and a history of hysterectomy, ovariectomy and menopausal hormonal treatments. Self-reported height and weight were used to calculate BMI, defined as weight $(\mathrm{kg})$ divided by height squared $\left(\mathrm{m}^{2}\right)$. In this cohort, self-reported anthropometry is reliable (in a sub-study the correlation coefficient between self-reported and measured height was $0.89 \mathrm{~m}^{2}$ and for weight was $0.94 \mathrm{~kg})^{(12)}$. We assessed usual physical activity with a questionnaire that included items on weekly hours spent walking, cycling, performing light and heavy household chores and recreational activities (e.g. swimming, tennis) during the summer and the winter. This questionnaire performed well in a previous study $^{(13)}$.

\section{Ascertainment of type 2 diabetes}

As previously described ${ }^{(14)}$, for case identification, we used selfreports, supplementary questionnaires and drug reimbursement information. Between 1993 and 2008, 2597 self-reported incident cases were confirmed either by a supplementary questionnaire or by drug reimbursement claims' database, and an additional 843 incident cases only through this database, but validated by a supplementary questionnaire. The present analysis is based on incident cases of diabetes after applying the exclusion criteria detailed above (no date of diagnosis, unavailable dietary information or unrealistic energy consumption, no follow-up, prevalent cancer or CVD and a diet for diabetes at baseline).

\section{Statistical analysis}

Nutrients were energy-adjusted using the residual method ${ }^{(15)}$. We categorised egg consumption as $0,<1,1-1.9,2-4.9$ and $\geq 5$ eggs/week, and cholesterol in quintiles, and used the lowest category as the referent. To test for trend, we used the median value for each category as a continuous variable. We evaluated cholesterol consumption using a substitution model for carbohydrates that included intakes of energy, saturated fat, unsaturated fat and protein (excluding carbohydrates) in the model. We calculated person-time from the date of completion of the dietary questionnaire to the date of diagnosis of diabetes, last follow-up available or June 2008. Hazard ratios (HR) and 95\% CI were estimated from Cox regression models with age as the time scale (SAS 9.1; SAS Institute Inc.). Models were adjusted for education, menopause, menopausal hormone therapy, treated hypertension and hypercholesterolaemia, physical activity, smoking, total energy, alcohol, processed red meat, coffee, fruit, vegetables, sugar and artificially sweetened beverages and BMI. We hypothesised that BMI may modify the association between dietary variables and diabetes; therefore, we tested for statistical interaction by including a cross-product term of median egg and cholesterol consumption by BMI $\left(<25, \geq 25 \mathrm{~kg} / \mathrm{m}^{2}\right)$ in the Cox model and by performing a log-likelihood ratio test.

\section{Results}

Mean age was 52.7 (SD 6.6) years; the prevalence of overweight was $15.5 \%$ and that of obesity was $3.2 \%$. Mean total egg consumption was 3.5 (SD 2.8) eggs/week, and the mean weekly hardboiled and omelette/mixed-dish egg intakes were 1.0 (SD 1.5 ) and 2.5 (SD 2.1), respectively. Mean cholesterol intake was $378(\mathrm{sD} 104) \mathrm{mg} / \mathrm{d}$, and the main food contributors of dietary cholesterol were omelette/mixed-dish egg (17\%), hardboiled egg (7\%), butter ( $4 \%)$, liver ( $4 \%)$, pizza and quiche (3\%), poultry (3\%), creams, dairy dessert and ice creams (3\%), beef $(3 \%)$ and cheese $(3 \%)$. The correlation coefficient for dietary cholesterol with saturated fat was 0.24 ( $P$ value $<0.0001)$ and $-0.26(P$ value $<0.0001)$ with dietary fibre. Egg consumption and cholesterol intake were directly related to intake of alcohol, processed red meat and coffee and to BMI and hypertension. We observed an inverse relation with treated hypercholesterolaemia and education (Table 1).

We identified 1803 incident diabetes cases during the study period. After multivariable adjustment, the HR comparing women who consumed $\geq 5$ eggs/week with non-consumers was $1.00(95 \%$ CI $0.78,1.29)$, with a $P_{\text {trend }}=0.11$ (Table 2$)$. The corresponding values for hardboiled egg consumption 
Table 1. Age-standardised risk factors for diabetes risk by total egg consumption and dietary cholesterol quintiles in a cohort of French women in 1993 (Mean values and standard deviations; percentages)

\begin{tabular}{|c|c|c|c|c|c|c|c|c|c|c|c|c|c|c|c|c|c|c|c|c|}
\hline & & & & & & & & & & & \multicolumn{10}{|c|}{ Quintiles of dietary cholesterol (mg/d) } \\
\hline & \multicolumn{10}{|c|}{ Eggs/week } & \multicolumn{2}{|l|}{1} & \multicolumn{2}{|l|}{2} & \multicolumn{2}{|l|}{3} & \multirow{2}{*}{$\frac{4}{393-453}$} & \multicolumn{3}{|c|}{5} \\
\hline & \multicolumn{2}{|l|}{0} & \multicolumn{2}{|l|}{$<1$} & \multicolumn{2}{|l|}{$1-1.9$} & \multicolumn{2}{|l|}{$2-4.9$} & \multicolumn{2}{|l|}{$\geq 5$} & $<294$ & & 294-345 & & \multicolumn{2}{|l|}{$346-392$} & & \multicolumn{3}{|c|}{$\geq 454$} \\
\hline Current smoker (\%) & 13.8 & & $13 \cdot 2$ & & $12 \cdot 8$ & & $13 \cdot 2$ & & $15 \cdot 5$ & & $12 \cdot 0$ & & $12 \cdot 6$ & & $12 \cdot 8$ & & $13 \cdot 7$ & & $16 \cdot 7$ & \\
\hline \multicolumn{21}{|l|}{$\mathrm{BMI}\left(\mathrm{kg} / \mathrm{m}^{2}\right)$} \\
\hline Mean & $22 \cdot 1$ & & $22 \cdot 3$ & & 22.5 & & $22 \cdot 9$ & & 23.4 & & $22 \cdot 1$ & & 22.5 & & $22 \cdot 8$ & & $23 \cdot 1$ & & $23 \cdot 6$ & \\
\hline SD & 2.7 & & $2 \cdot 8$ & & $2 \cdot 9$ & & $3 \cdot 1$ & & 3.4 & & $2 \cdot 7$ & & $2 \cdot 9$ & & 3.0 & & 3.1 & & 3.5 & \\
\hline Treated hypertension (\%) & 7.5 & & 7.5 & & 7.6 & & 7.8 & & 8.3 & & $7 \cdot 0$ & & 7.5 & & $7 \cdot 6$ & & 8.1 & & 8.8 & \\
\hline Hypercholesterolaemia (\%) & 11.3 & & 8.0 & & $6 \cdot 4$ & & $5 \cdot 1$ & & 4.3 & & 7.7 & & $6 \cdot 3$ & & $5 \cdot 1$ & & $5 \cdot 2$ & & 4.7 & \\
\hline Current use of MHT (\%) & $22 \cdot 1$ & & 24.5 & & 23.4 & & 23.5 & & $23 \cdot 3$ & & $22 \cdot 8$ & & $23 \cdot 4$ & & $23 \cdot 9$ & & 23.9 & & 23.6 & \\
\hline \multicolumn{21}{|l|}{ Physical activity (h/week) } \\
\hline Mean & $6 \cdot 4$ & & $6 \cdot 2$ & & $6 \cdot 2$ & & 6.4 & & 6.8 & & 6.4 & & $6 \cdot 3$ & & 6.5 & & 6.4 & & 6.6 & \\
\hline SD & 8.4 & & $9 \cdot 0$ & & 7.7 & & 7.8 & & 8.1 & & $8 \cdot 3$ & & $7 \cdot 3$ & & $8 \cdot 1$ & & $7 \cdot 7$ & & 8.3 & \\
\hline University education (\%) & $40 \cdot 8$ & & 40.5 & & 38.1 & & $35 \cdot 0$ & & $32 \cdot 4$ & & $38 \cdot 4$ & & $37 \cdot 1$ & & $36 \cdot 1$ & & $34 \cdot 8$ & & 33.6 & \\
\hline Mean dietary intake & Mean & SD & Mean & SD & Mean & SD & Mean & SD & Mean & SD & Mean & SD & Mean & SD & Mean & SD & Mean & SD & Mean & SD \\
\hline Total energy $(\mathrm{kJ} / \mathrm{d})$ & 7909 & 2149 & 8108 & 1988 & 8453 & 2026 & 9048 & 2155 & 9814 & 2438 & 8848 & 2552 & 9073 & 2320 & 9077 & 2192 & 8969 & 2107 & 8700 & 2026 \\
\hline Processed meat (servings/d) & 0.3 & 0.4 & 0.3 & 0.3 & 0.4 & 0.3 & 0.5 & 0.4 & 0.6 & 0.5 & 0.3 & 0.3 & 0.4 & 0.3 & 0.5 & 0.4 & 0.6 & 0.4 & 0.6 & 0.4 \\
\hline Fruits (servings/d) & 2.6 & 1.8 & 2.5 & 1.6 & 2.5 & 1.6 & 2.5 & 1.6 & 2.6 & 1.8 & $3 \cdot 1$ & 1.9 & 2.7 & 1.7 & 2.5 & 1.6 & $2 \cdot 3$ & 1.5 & $2 \cdot 1$ & 1.5 \\
\hline Vegetables (servings/d) & $2 \cdot 3$ & 1.3 & $2 \cdot 2$ & 1.2 & $2 \cdot 2$ & 1.2 & $2 \cdot 3$ & 1.2 & $2 \cdot 4$ & 1.3 & $2 \cdot 4$ & 1.3 & $2 \cdot 3$ & 1.2 & $2 \cdot 2$ & $1 \cdot 2$ & 2.3 & 1.2 & 2.3 & 1.3 \\
\hline Coffee (cups/d) & $2 \cdot 2$ & $2 \cdot 2$ & $2 \cdot 1$ & $2 \cdot 0$ & $2 \cdot 2$ & $2 \cdot 0$ & $2 \cdot 3$ & $2 \cdot 1$ & 2.6 & $2 \cdot 3$ & $2 \cdot 1$ & $2 \cdot 1$ & $2 \cdot 2$ & 2.0 & $2 \cdot 3$ & $2 \cdot 1$ & $2 \cdot 4$ & $2 \cdot 1$ & 2.6 & $2 \cdot 2$ \\
\hline Sugar-sweetened drinks (per week) & 7.6 & 37.8 & $6 \cdot 2$ & 33.8 & $7 \cdot 0$ & $32 \cdot 2$ & 7.6 & $30 \cdot 0$ & $9 \cdot 2$ & 36.3 & $7 \cdot 3$ & 35.5 & $8 \cdot 2$ & 34.7 & 7.9 & $30 \cdot 2$ & 7.9 & 31.1 & 7.1 & $31 \cdot 3$ \\
\hline Diet drinks (per week) & $2 \cdot 9$ & $24 \cdot 1$ & 1.9 & 20.4 & $2 \cdot 4$ & 25.9 & $2 \cdot 4$ & 24.2 & 4.5 & 37.7 & $2 \cdot 2$ & 24.6 & $2 \cdot 3$ & 24.1 & $2 \cdot 3$ & 23.5 & $2 \cdot 8$ & $26 \cdot 3$ & 4.4 & 36.5 \\
\hline Alcohol (g/d) & 9.5 & $13 \cdot 1$ & $10 \cdot 0$ & $13 \cdot 1$ & $10 \cdot 6$ & $12 \cdot 7$ & 11.9 & 13.7 & $13 \cdot 2$ & $\quad 15.3$ & 8.9 & $11 \cdot 6$ & $10 \cdot 7$ & $12 \cdot 7$ & 11.6 & 13.4 & $12 \cdot 6$ & $14 \cdot 3$ & 14.1 & $16 \cdot 1$ \\
\hline Cholesterol (mg/d) & 247 & 98 & 276 & 88 & 320 & 93 & 395 & 104 & 540 & 145 & - & - & - & & - & & - & & - & - \\
\hline
\end{tabular}

MHT, menopausal hormone therapy 
Table 2. Age-adjusted and multivariable-adjusted hazard ratios (HR) of type 2 diabetes according to the number of eggs consumed per week in the Etude Epidémiologique auprès des femmes de la Mutuelle Générale de l'Education Nationale cohort study (1993-2008)

(Hazard ratios and $95 \%$ confidence intervals)

\begin{tabular}{|c|c|c|c|c|c|c|c|c|c|c|}
\hline & \multicolumn{9}{|c|}{ Eggs/week } & \multirow[b]{3}{*}{$P_{\text {trend }}$} \\
\hline & \multirow[b]{2}{*}{0} & \multicolumn{2}{|c|}{$0.1-0.9$} & \multicolumn{2}{|c|}{$1-1.9$} & \multicolumn{2}{|c|}{$2-4.9$} & \multicolumn{2}{|c|}{$\geq 5$} & \\
\hline & & $\mathrm{HR}$ & $95 \% \mathrm{Cl}$ & $\mathrm{HR}$ & $95 \% \mathrm{Cl}$ & $\mathrm{HR}$ & $95 \% \mathrm{Cl}$ & $\mathrm{HR}$ & $95 \% \mathrm{Cl}$ & \\
\hline \multicolumn{11}{|l|}{ Total egg consumption } \\
\hline Median intake & 0 & 0.7 & & 1.7 & & 3.4 & & 6.9 & & \\
\hline Number of cases & 76 & 153 & & 317 & & 770 & & 487 & & \\
\hline Person-years & 40152 & \multicolumn{2}{|c|}{99051} & \multicolumn{2}{|c|}{177678} & \multicolumn{2}{|c|}{385945} & \multicolumn{2}{|c|}{176307} & \\
\hline Age-adjusted & Ref. & 0.84 & $0.64,1 \cdot 10$ & 1.01 & $0.79,1.30$ & 1.17 & $0.92,1.48$ & 1.58 & $1.23,2.02$ & $<0.0001$ \\
\hline Multivariable ${ }^{*}$ & Ref. & 0.86 & $0.65,1.14$ & 1.04 & $0.81,1.34$ & 1.20 & $0.94,1.52$ & 1.56 & $1.22,1.99$ & $<0.0001$ \\
\hline + Diet† & Ref. & 0.90 & $0.68,1.19$ & 1.06 & $0.82,1.36$ & 1.17 & $0.92,1.48$ & 1.42 & $1.11,1.83$ & $<0.0001$ \\
\hline + Diet† + BMI & Ref. & 0.84 & $0.64,1.11$ & 0.91 & $0.71,1.17$ & 0.94 & $0.74,1.20$ & 1.00 & $0.78,1.29$ & 0.11 \\
\hline \multicolumn{11}{|l|}{ Hardboiled eggs } \\
\hline Median intake & 0 & 0.5 & & 1.5 & & $3 \cdot 0$ & & $7 \cdot 0$ & & \\
\hline Number of cases & 481 & 644 & & 356 & & 252 & & 70 & & \\
\hline Person-years & 275240 & \multicolumn{2}{|c|}{347858} & \multicolumn{2}{|c|}{147855} & \multicolumn{2}{|c|}{87954} & \multicolumn{2}{|c|}{20226} & \\
\hline Age-adjusted & Ref. & $1 \cdot 10$ & $0.97,1.24$ & 1.43 & $1.24,1.64$ & 1.67 & $1.43,1.95$ & 1.95 & $1.52,2.51$ & $<0.0001$ \\
\hline Multivariable* & Ref. & 1.08 & $0.96,1.21$ & 1.36 & $1.18,1.56$ & 1.53 & $1.31,1.79$ & 1.74 & $1 \cdot 35,2 \cdot 25$ & $<0.0001$ \\
\hline + Diet† & Ref. & 1.05 & $0.93,1.19$ & 1.26 & $1.09,1.45$ & 1.37 & $1.17,1.60$ & 1.51 & $1.17,1.95$ & $<0.0001$ \\
\hline + Diet† + omelette/mixed-dish eggs & Ref. & 1.05 & $0.93,1.18$ & 1.22 & $1.06,1.41$ & 1.31 & $1.12,1.54$ & 1.45 & $1.11,1.87$ & $<0.0001$ \\
\hline + Diet† + omelette/mixed-dish eggs + BMI & Ref. & 1.04 & $0.92,1.18$ & 1.13 & $0.98,1.30$ & 1.13 & $0.96,1.30$ & 1.16 & $0.89,1.50$ & 0.12 \\
\hline \multicolumn{11}{|l|}{ Omelette/mixed-dish eggs } \\
\hline Median intake & 0 & 0.9 & & 1.9 & & 3.4 & & $6 \cdot 0$ & & \\
\hline Number of cases & 143 & 274 & & 500 & & 679 & & 207 & & \\
\hline Person-years & 69786 & \multicolumn{2}{|c|}{169868} & \multicolumn{2}{|c|}{259430} & \multicolumn{2}{|c|}{298736} & \multicolumn{2}{|c|}{81313} & \\
\hline Age-adjusted & Ref. & 0.81 & $0.66,1.00$ & 1.00 & $0.83,1.21$ & 1.21 & $1.00,1.45$ & 1.29 & $1.04,1.60$ & $<0.0001$ \\
\hline Multivariable* & Ref. & 0.84 & $0.69,1.03$ & 1.04 & $0.86,1.25$ & 1.24 & $1.03,1.49$ & 1.32 & $1.06,1.64$ & $<0.0001$ \\
\hline+ Diet† & Ref. & 0.87 & $0.71,1.06$ & 1.04 & $0.86,1.26$ & 1.19 & $0.99,1.44$ & 1.22 & $0.98,1.52$ & $<0.0001$ \\
\hline + Diet† + hardboiled eggs & Ref. & 0.86 & $0.70,1.06$ & 1.02 & $0.85,1.24$ & 1.15 & $0.95,1.38$ & $1 \cdot 13$ & $0.91,1.42$ & 0.002 \\
\hline + Diet† + hardboiled eggs + BMI & Ref. & 0.85 & $0.69,1.04$ & 0.92 & $0.69,1.04$ & 0.98 & $0.81,1.18$ & 0.91 & $0.73,1.14$ & 0.67 \\
\hline
\end{tabular}

Ref., referent values.

* Adjusted for education, smoking (never, secondhand smoke, past, current), physical activity (h/week, quartiles), hormone replacement therapy (premenopausal, current, past, never), hypertension, hypercholesterolaemia and energy (quintiles).

† Additional adjustment alcohol (quintiles), processed red meat (quintiles), coffee (quintiles), fruits (quintiles), vegetables (quintiles), sugar-sweetened drinks (no consumers +quartiles) and artificially sweetened drinks (no consumers + quartiles). 
Eggs, cholesterol and incident diabetes

were HR 1.16 (95\% CI 0.89, 1.50; $\left.P_{\text {trend }}=0.12\right)$. For omelette/ mixed-dish eggs these values were HR 0.91 (95\% CI 0.73, 1.14; $\left.P_{\text {trend }}=0.67\right)$. For dietary cholesterol, women in the highest quintile of intake (median, $507 \mathrm{mg}$ ) had a $40 \%$ higher rate of diabetes as compared with those in the lowest quintile (median, $241 \mathrm{mg}$ ) (HR 1.40; 95\%CI 1.19, 1.63; $P_{\text {trend }}<0.0001$ ) (Table 3). There was an attenuation when results were adjusted for BMI; however, the relations remained statistically significant. In the substitution model, each additional $100 \mathrm{mg}$ dietary cholesterol per $4184 \mathrm{~kJ}$ (or $1000 \mathrm{kcal}$ ) was associated with a $14 \%$ increase in the risk for diabetes (HR 1.14; $95 \%$ CI 1.02, 1.26). These associations did not differ between normal weight $\left(\mathrm{BMI}<25 \mathrm{~kg} / \mathrm{m}^{2}\right.$ ) and overweight individuals (BMI $\geq 25 \mathrm{~kg} / \mathrm{m}^{2}$ ). The $P$ values for interaction were 0.36 for egg consumption and $0 \cdot 11$ for cholesterol intake. In sensitivity analyses, we stratified models on hypertension and hypercholesterolaemia. The association remained in hypertensive and non-hypertensive women. We observed a slightly stronger association among hypertensive women (comparing extreme quintiles: $\mathrm{HR}_{\text {hypertensive }}=1 \cdot 58 ; 95 \% \mathrm{CI} 1 \cdot 11,2 \cdot 26, v$. $\mathrm{HR}_{\text {non-hypertensive }}=$ $1.38 ; 95 \%$ CI $1.15,1.65)$; however, there was no evidence of heterogeneity $\left(P_{\text {interaction }}=0 \cdot 91\right)$. For hypercholesterolaemia, there was no statistical evidence of a difference in the association of dietary cholesterol with diabetes. HR for the comparison of extreme categories of dietary cholesterol were 1.19 (95\% CI 0.76, 1.86; $\left.P_{\text {trend }}=0.24\right)$ and 1.44 (95\% CI 1.21 , $\left.1.71 ; P_{\text {trend }}<0.0001\right)$ in women with and without hypercholesterolaemia, respectively $\left(P_{\text {interaction }}=0 \cdot 21\right)$.

\section{Discussion}

After a median 13.8-year follow-up, dietary cholesterol was found to be associated with a risk for type 2 diabetes in a prospective study on French women. We found no overall association with egg consumption.

Animal studies suggest that diets rich in $\mathrm{fat}^{(16)}$ and egg consumption $^{(2)}$ may disrupt glucose metabolism and increase circulating insulin. In addition, data from different sources suggest that the cholesterol metabolism may contribute to diabetes risk ${ }^{(17)}$. Our results confirm a previous observation of a $36 \%$ higher diabetes risk in a large cohort of US women on comparing women with the highest and the lowest dietary cholesterol intakes ${ }^{(3)}$. In a study that included a prospective cohort and a case-control study of maternal diet, there was a more than 2-fold higher risk for gestational diabetes on comparing extreme categories of dietary cholesterol ${ }^{(1)}$. However, in a cohort study in Japan, dietary cholesterol was not associated with diabetes incidence after a 5 -year follow-up ${ }^{(10)}$. In contrast to Western populations, in this study, red meat was not the most common food source for dietary cholesterol. Therefore, it is possible that the discrepancy may be due to differences in the distribution of cholesterol food sources. In E3N, we previously observed a relation between processed red meat and diabetes $^{(18)}$; thus, we adjusted for processed red meat in our models. In addition, it is possible that we may have been able to detect an association because the distribution of cholesterol was wider in our study. The mean cholesterol intake in women in Japan for the highest category was $390 \mathrm{mg} / \mathrm{d}$, whereas in the 
present study the median intake for the highest quintile of intake was $507 \mathrm{mg} / \mathrm{d}$. A recent analysis in the smaller Kuopio prospective cohort did not show an association between cholesterol intake, assessed using 4-d food records and incident diabetes after a 19-year follow-up ${ }^{(9)}$. Individuals may have changed their diet over the follow-up period, potentially attenuating the association. In a sensitivity analysis restricted to the first 10 years of follow-up, cholesterol intake appeared to confer risk of diabetes; however, results were not statistically significant. For egg consumption, our results differ from observations reported in a meta-analysis based on four prospective US studies ${ }^{(19)}$ that found an association between egg consumption and type 2 diabetes and also from a study on maternal diet and gestational diabetes ${ }^{(1)}$. More recently, this association was evaluated in a small Spanish cohort study with relatively few incident cases of diabetes ${ }^{(8)}$ and in the Kuopio study in Finland ${ }^{(9)}$; no association was observed in either study. Eggs are often consumed with other foods, and there are several methods of preparation that include the addition of other nutrients. Thus, other foods or added nutrients may be responsible for the observed relation. In our study, we did not observe a relation between egg consumption and diabetes risk after adjustment for BMI, a variable that is known to be strongly associated with diabetes and is also associated with diet.

The prospective design, limited loss to follow-up, use of a validated dietary questionnaire and the possibility of evaluating different egg preparations separately are important strengths of the present analysis. There are some limitations to consider. First and foremost, there is some evidence for potential biological mechanisms supporting altered cholesterol metabolism and diabetes risk. However, it is less clear how dietary cholesterol could affect diabetes occurrence. Diet was assessed only at baseline, and participants may have changed their diet during follow-up. Thus, a non-differential measurement error is possible and could potentially explain null results. Other dietary analyses in this cohort have shown significant associations with diabetes and could be indicative that this error may not be substantial. Non-differential misclassification of diabetes is also a possibility. However, assuming very few false positives, this error probably had no measurable effect on our estimates. Confounding by unmeasured factors such as dietary factors prior to baseline, or by variables that are difficult to measure, such as unhealthy behaviour, can never be ruled out. We adjusted for the most important risk factors for diabetes and saw a large attenuation of HR. We further explored this possibility by stratifying analyses on hypercholesterolaemia and hypertension, two diagnoses that could be related to unhealthy behaviours and could also alter dietary habits. However, the association between dietary cholesterol and diabetes remained among individuals without these diagnoses.

Our results suggest that dietary cholesterol is associated with type 2 diabetes, but habitual egg consumption is not. These results should be interpreted with caution as the underlying mechanism for the relation of dietary cholesterol and diabetes is unclear, and there is potential for residual confounding. Future studies should address potential mechanisms by which glucose metabolism may be disrupted by cholesterol intake.

\section{Acknowledgements}

The authors are indebted to the participants in the Etude Epidémiologique auprès des femmes de la Mutuelle Générale de l'Education Nationale (E3N) for their continuing dedication and support. The authors thank Thierno Mamadou Bah for additional statistical support.

The E3N cohort study was carried out with the financial support of the Mutuelle Générale de l'Education Nationale, European Community, French League against Cancer, Gustave Roussy Institute and French Institute of Health and Medical Research. The validation of potential diabetes cases was supported by the European Union (Integrated Project LSHM-CT-2006-037197 in the Framework Programme 6 of the European Community) InterAct project. The present analysis was conducted with the support of the International Associated Laboratory in Nutrition, Obesity, Hormones and Cardiometabolic Disease and Depression, an ongoing collaboration between the National Institute of Health and Medical Research (Inserm, France) and the National Institute of Public Health (INSP, Mexico). M. L. is a Bernard Lown Visiting Scholar in Cardiovascular Health at the Harvard School of Public Health.

M. L. formulated the research question. M. L., A. B., M.-C. B.-R. and F. C.-C. designed the study; M. L., A. B., M.-C. B.-R. and G. F. conducted research; A. B. analysed the data; M. L., B. B. and M.-C. B.-R. and F. C.-C. wrote the paper; M. L. had primary responsibility for final content. All authors read and approved the final manuscript.

There are no conflicts of interest.

\section{References}

1. Qiu C, Frederick IO, Zhang C, et al. (2011) Risk of gestational diabetes mellitus in relation to maternal egg and cholesterol intake. Am J Epidemiol 173, 649-658.

2. Adamopoulos PN, Papamichael CM, Zampelas A, et al. (1996) Cholesterol and unsaturated fat diets influence lipid and glucose concentrations in rats. Comp Biochem Physiolo $B$ Biochem Mol Biol 113, 659-663.

3. Salmeron J, Hu FB, Manson JE, et al. (2001) Dietary fat intake and risk of type 2 diabetes in women. Am J Clin Nutr 73, 1019-1026.

4. Bowers K, Tobias DK, Yeung E, et al. (2012) A prospective study of prepregnancy dietary fat intake and risk of gestational diabetes. Am J Clin Nutr 95, 446-453.

5. Djousse L, Gaziano JM, Buring JE, et al. (2009) Egg consumption and risk of type 2 diabetes in men and women. Diabetes Care 32, 295-300.

6. Vang A, Singh PN, Lee JW, et al. (2008) Meats, processed meats, obesity, weight gain and occurrence of diabetes among adults: findings from Adventist Health Studies. Ann Nutr Metab 52, 96-104.

7. Djousse L, Kamineni A, Nelson TL, et al. (2010) Egg consumption and risk of type 2 diabetes in older adults. $\mathrm{Am} \mathrm{J}$ Clin Nutr 92, 422-427.

8. Zazpe I, Beunza JJ, Bes-Rastrollo M, et al. (2013) Egg consumption and risk of type 2 diabetes in a Mediterranean cohort; the Sun project. Nutr Hosp 28, 105-111.

9. Virtanen JK, Mursu J, Tuomainen TP, et al. (2015) Egg consumption and risk of incident type 2 diabetes in men: the Kuopio Ischaemic Heart Disease Risk Factor Study. Am J Clin Nutr 101, 1088-1096. 
10. Kurotani K, Nanri A, Goto A, et al. (2014) Cholesterol and egg intakes and the risk of type 2 diabetes: the Japan Public Health Center-based Prospective Study. Br J Nutr 112, 1636-1643.

11. van Liere MJ, Lucas F, Clavel F, et al. (1997) Relative validity and reproducibility of a French dietary history questionnaire. Int J Epidemiol 26, Suppl. 1, S128-S136.

12. Tehard B, van Liere MJ, Com Nougue C, et al. (2002) Anthropometric measurements and body silhouette of women: validity and perception. I Am Diet Assoc 102, 1779-1784.

13. Tehard B, Friedenreich CM, Oppert JM, et al. (2006) Effect of physical activity on women at increased risk of breast cancer: results from the $\mathrm{E} 3 \mathrm{~N}$ cohort study. Cancer Epidemiol Biomarkers Prev 15, 57-64.

14. Lajous M, Bijon A, Fagherazzi G, et al. (2014) Body mass index, diabetes, and mortality in french women: explaining away a 'paradox'. Epidemiology 25, 10-14.
15. Willett W (1998) Nutritional Epidemiology, 2nd ed. vol. 30, Monographs in Epidemiology and Biostatistics. New York, NY: Oxford University Press.

16. Wu L, Vikramadithyan R, Yu S, et al. (2006) Addition of dietary fat to cholesterol in the diets of LDL receptor knockout mice: effects on plasma insulin, lipoproteins, and atherosclerosis. J Lipid Res $\mathbf{4 7}, 2215-2222$.

17. Brunham LR, Kruit JK, Verchere CB, et al. (2008) Cholesterol in islet dysfunction and type 2 diabetes. J Clin Invest $\mathbf{1 1 8}$, 403-408.

18. Lajous M, Tondeur L, Fagherazzi G, et al. (2012) Processed and unprocessed red meat consumption and incident type 2 diabetes among French women. Diabetes Care 35, 128-130.

19. Shin JY, Xun P, Nakamura Y, et al. (2013) Egg consumption in relation to risk of cardiovascular disease and diabetes: a systematic review and meta-analysis. Am J Clin Nutr 98, 146-159. 